\title{
Learning to Select Useful Landmarks
}

\author{
Russell Greiner \\ Siemens Corporate Research \\ Princeton, NJ 08540 \\ greiner@learning.siemens.com
}

\author{
Ramana Isukapalli \\ Department of Computer Science \\ Rutgers University \\ ramana@cs.rutgers.edu
}

\begin{abstract}
To navigate effectively, an autonomous agent must be able to quickly and accurately determine its current location. Given an initial estimate of its position (perhaps based on dead-reckoning) and an image taken of a known environment, our agent first attempts to locate a set of landmarks (real-world objects at known locations), then uses their angular separation to obtain an improved estimate of its current position. Unfortunately, some landmarks may not be visible, or worse, may be confused with other landmarks, resulting in both time wasted in searching for invisible landmarks, and in further errors in the agent's estimate of its position. To address these problems, we propose a method that uses previous experiences to learn a selection function that, given the set of landmarks that might be visible, returns the subset which can reliably be found correctly, and so provide an accurate registration of the agent's position. We use statistical techniques to prove that the learned selection function is, with high probability, effectively at a local optimal in the space of such functions. This report also presents empirical evidence, using real-world data, that demonstrate the effectiveness of our approach.
\end{abstract}

\section{Introduction}

To navigate effectively, an autonomous agent $R$ must be able to quickly and accurately determine its current location. $R$ can obtain fairly accurate estimates of its position using dead-reckoning; unfortunately, the errors in these estimates accumulate over long distances, which can lead to unacceptable performance (read "bumping into walls" or "locating the wrong office"). An obvious way to reduce this problem is to observe the environment, and use the information in these observations to improve our estimate of $R$ 's position; cf., the work using Kalman filters (Kosaka \& Kak 1992; Cox \& Wilfong 1990) and other techniques (Smith \& Cheeseman 1987; Kuipers \& Levitt 1988; Fennema et al. 1990; Engelson 1992) We will model the environment using only a set of "landmarks", each a (potentially visible) real-world object at a known location; these objects could be doors, corners and pictures when specifying the hallways within building, or major buildings, junctions and prominent signs when specifying the streets within a city. ${ }^{1}$ Given an initial estimate of its position (perhaps based on dead-reckoning) and an image taken of a known environment, $R$ can first attempt to locate a set of possibly visible landmarks, then use their angular separation to obtain an improved estimate of its current position.

Landmark-based position estimation is a popular technique in robot navigation (Case 1986; Sugihara 1988; 1987; Levitt \& Lawton 1990). Many of these landmark-based methods assume that all landmarks can be found reliably. Unfortunately, some landmarks may not be visible; for example, certain corners may always be in shadow and so are difficult to see, or some hanging pictures may have been removed after the floor-plan was released. These can force $R$ to waste time, searching in vain for invisible landmarks. Worse, some landmarks may be easily confused with others; e.g., door $A$ may be mistaken for door $B$, or some landmark $A$ (say the convex corner of two walls) may be occluded by another object $B$ (say the convex corner of filing cabinet) that looks sufficiently similar that $R$ might think that $B$ is $A$. As this can cause $R$ to believe that $A$ is located at $B$ 's position, these mis-identified objects can produce further errors in $R$ 's estimate of its position. ${ }^{2}$

It therefore makes sense to search for only the subset of the potentially visible landmarks that can be found

\footnotetext{
${ }^{1}$ Notice this information is essentially the same as the information required for the navigation task itself, to specify the destination or some required intermediate points. $N . b$., we assume that this set of all possible landmarks is known initially; this contrasts with other systems that also attempt to learn the set of landmarks from the observations; cf., (Kuipers \& Byun 1988) and others.

${ }^{2}$ Another possible complication is that $R$ may identify a wide landmark correctly, but mistakenly refer to the wrong position within that landmark. Also, $R$ uses a set of identified landmarks to locate its position; depending on the geometric positions of these landmarks, small errors in landmark location may lead to quite large errors in $R$ 's positional estimate. We of course prefer landmarks sets that provide position estimates that are relatively insensitive to errors in landmark identification.
} 
reliably, which are not confusable with others, etc. Unfortunately, it can be very difficult to determine this good subset a priori, as (1) the landmarks that are good for one set of $R$-positions can be bad for another; (2) the decision to seek a landmark can depend on many difficult-to-incorporate factors, such as lighting conditions and building shape; and (3) the reliability of a landmark can also depend on unpredictable events; e.g., exactly where $R$ happens to be when it observes its environment, how the building has changed after the floor-plan was finalized, and whether objects (perhaps people) are moving around the area where $R$ is looking. These factors make it difficult, if not impossible, to designate the set of good landmarks ahead of time.

This report presents a way around this problem: Section 2 proposes a method that learns a good "selection function" that, given the set of landmarks that may be visible, returns the subset which can usually be found correctly. We also use statistical techniques to prove that this learned selection function is, with high probability, effectively at a local optimum in the space of such functions. Section 3 then presents empirical results that demonstrate that this algorithm can work effectively. We first close this section by presenting a more precise description of the performance task, showing how $R$ estimates its position:

Specification of Performance Task: At each point, $R$ will have an estimate $\hat{\mathrm{x}}$ of its current position $x$ and a measure of the uncertainty (here the covariance matrix). $R$ uses the LMs ( $\mathbf{x}$ ) algorithm to specify the subset of the landmarks that may be visible from each position $\mathbf{x}$; we assume LMs ( $\hat{\mathbf{x}})$ is essentially the same as LMs $(x)$. (I.e., we assume that $R$ 's estimate of its position is sufficient to specify a good approximation of the set of possibly appropriate landmarks.) $R$ also uses an algorithm Locate ( $\hat{\mathbf{x}}, \hat{\sigma}$, img, lms ) that, given $R$ 's estimate of its position $\hat{\mathbf{x}}$ and uncertainty $\hat{\sigma}$, an image img taken at $R$ 's current position and a set of landmarks Ims, returns a new estimated position (and uncertainty) for $R$.

To instantiate these processes: In the current RATBOT system (Hancock \& Judd 1993), the LMs process uses a comprehensive "landmark-description" of the environment, which is a complete list of all of the objects in that environment that could be visible, together with their respective positions. This could be based on the floor-plan of a building, which specifies the positions of the building's doors, walls, wallhangings, etc.; or in another context, it could be a map of the roads of a city, which specifies the locations of the significant buildings, signs, and so forth. The Locate ( $\hat{\mathbf{x}}, \hat{\sigma}$, img, Ims ) process first attempts to find each landmark $l_{i} \in$ lms within the image img; here it uses $\hat{x}$ and $\hat{\sigma}$ to specify where in the image to look for this $l_{i}$. It will find a subset of these landmarks, each at some angle (relative to a reference landmark). Locate then uses simple geometric reasoning to obtain a set of new estimates of $R$ 's position; perhaps one from each set of three found landmarks (Hancock \& Judd 1994), or see (Gurvits \& Betke 1994). After removing the obvious outliers, Locate returns the centroid of the remaining estimates as its positional-estimate for $R$, and the variance of these estimates as the measure of uncertainty; see (Hancock \& Judd 1993).

As our goal is an efficient way of locating $R$ 's position, our implementation uses an inexpensive way of finding the set of landmarks based on simple tests on the visual image; $n . b$. , we are not using a general vision system, which would attempt to actually identify specific objects and specify particular qualities from the visual information. ${ }^{3}$

\section{Function for Selecting Good Landmarks}

While many navigation systems would attempt to locate all of the landmarks that might be visible in an image (i.e., the full set returned by $\operatorname{LMs}(\hat{\mathbf{x}})$ ), we argued above that it may be better to seek only a subset of these landmarks: By avoiding "problematic" landmarks (e.g., ones that tend to be not visible, or confusable), $R$ may be able to obtain an estimate of its location more quickly, and moreover, possibly obtain an estimate that is more accurate.

We therefore want to identify and ignore these bad landmarks. We motivate our approach by first presenting two false leads: One immediate suggestion is to simply exclude the bad landmarks from the catalogue of all landmarks that LMs uses, meaning LMs (.) will never return certain landmarks. One obvious complication is the complexity of determining which landmarks are bad, as this can depend on many factors, including the color of the landmark, the overall arrangement of the entire environment (which would specify which landmarks could be occluded), the lighting conditions, etc. A more serious problem is the fact that a landmark that is hard to see from one $R$ position may be easy to see, and perhaps invaluable, from another; here, $R$ should be able to use that landmark when registering its location from some positions, but not from others.

We therefore decided to use, instead, a selection function Sel that filters out the bad landmarks from the set of possibly visible landmarks, $1 \mathrm{~ms}=\mathrm{LMs}($ $\hat{\mathbf{x}})$ : Here, each selection function $\mathrm{Sel}_{i}$ returns a subset $\operatorname{Sel}_{i}(1 \mathrm{~ms}, \hat{\mathrm{x}}, \hat{\sigma})=\operatorname{lms}_{i} \subseteq 1 \mathrm{~ms} ; R$ then uses this subset to compute its location, returning Locate $\left(\hat{\mathbf{x}}, \hat{\sigma}, \mathrm{img}, \mathrm{lms}_{i}\right)$. We want to use a selection function $\operatorname{Sel}_{i}$ such that Locate $\left(\hat{\mathbf{x}}, \hat{\sigma}\right.$, img, $\operatorname{lms}_{i}$ ) is reliably close to $R$ 's true position, $\mathbf{x}$. To make this

\footnotetext{
${ }^{3}$ Figure 3 shows, and describes, the actual "images" we use. Also, this articles does not provide pseudo-code for either LMs or Locate, as our learning algorithm regards these process as black-boxes.
} 
more precise, let

$$
\begin{aligned}
& \operatorname{Err}\left(\operatorname{Sel}_{i},\langle\mathbf{x}, \hat{\mathbf{x}}, \hat{\sigma}, \mathrm{img}\rangle\right)= \\
& \quad\left\|\mathbf{x}-\operatorname{Locate}\left(\hat{\mathbf{x}}, \hat{\sigma}, \operatorname{img}, \operatorname{Sel}_{i}(\operatorname{LMs}(\hat{\mathbf{x}}), \hat{\mathbf{x}}, \hat{\sigma})\right)\right\|
\end{aligned}
$$

be the error ${ }^{4}$ for the selection function $\mathrm{Sel}_{i}$ and any "situation" $\langle\mathbf{x}, \hat{\mathbf{x}}, \hat{\sigma}, \mathrm{img}\rangle$, and let

$$
\begin{array}{r}
\operatorname{AveErr}\left(\operatorname{Sel}_{i}\right)= \\
E_{\{\mathrm{x}, \hat{\mathrm{x}}, \hat{\sigma}, \mathrm{img}\rangle}\left[\operatorname{Err}\left(\operatorname{Sel}_{i},\langle\mathrm{x}, \hat{\mathbf{x}}, \hat{\sigma}, \mathrm{img}\rangle\right)\right]
\end{array}
$$

be the expected error, over the distribution of situations $\langle\mathbf{x}, \hat{\mathbf{x}}, \hat{\sigma}, \mathrm{img}\rangle$, where $E$. [.] is the expectation operator. Our goal is a selection function $\mathrm{Sel}_{o p t}$ that minimizes this expected value, over the set of possible selection functions.

The second false lead involves "engineering" this optimal selection function initially. One problem, as observed above, is the difficulty of determining "analytically" which landmarks are going to be problematic for any single situation. Worse, recall that our goal is to find the selection function that works best over the distribution of situations; which depends on the distribution of $R$ 's actual positions when the function is called, the actual intensity of light sources, what other objects have been moved where, etc. Unfortunately, this distribution of situations is not known a priori.

We are therefore following a third (successful) approach: of learning a good selection function. Hcrc, we first specify a large (and we hope, comprehensive) class of possible selection functions $\mathcal{S}=\left\{\operatorname{Sel}_{i}\right\}$. Then, given "labeled samples" - each consisting of $R$ 's position and uncertainty estimates, the relevant landmarkset and image, and as the label, $R$ 's actual position - identify the selection function $\mathrm{Sel}_{i}$ which minimizes $\operatorname{AveErr}\left(\mathrm{Sel}_{i}\right)$.

Space of Selection Function: We define each selection function $\mathrm{Sel}_{k} \in \mathcal{S}$ as a conjunction of its particular set of "heuristics" or "filters"; Filters(Select $\left.k_{i}\right)=$ $\left\{f_{1}, \ldots, f_{m}\right\}$, where each filter $f_{i}$ is a predicate that accepts some landmarks and rejects others. Hence, the Select $_{k}$ ( lms, $\hat{\mathbf{x}}, \hat{\sigma}$ ) procedure will examine each $\ell \in \mathrm{lms}$ individually, and reject it if any $f_{i}$ filter rejects it; see Figure 1.

While we can define a large set of such filters, this report focuses on only two parameterized filters:

$$
\begin{array}{rr}
\operatorname{BadType}_{K_{3}}(\ell, \hat{\mathbf{x}}, \hat{\sigma}): & \text { Reject } \ell \text { if Type( } \ell) \notin K_{3} \\
\text { TooSmall }_{k_{1}, k_{2}}(\ell, \hat{\mathbf{x}}, \hat{\sigma}): & \text { Reject } \ell \text { if }\|\operatorname{Posn}(\ell)-\hat{\mathbf{x}}\|>k_{1} \\
& \text { and AngleWidth }(\ell, \hat{\mathbf{x}})<k_{2}
\end{array}
$$

\footnotetext{
${ }^{4}$ As we are also considering the efficiency of the overall process, we will actually use the slightly more complicated error function presented in Section 3 below. This is also why we did not address the landmark-selection task using robust analysis: Under that approach, our system would first spend time and resources seeking each landmark, and would then decide whether to use each possible correspondence. As our approach, instead, specifies which landmarks should be sought, we will gather less data, and so expend fewer resources.
}

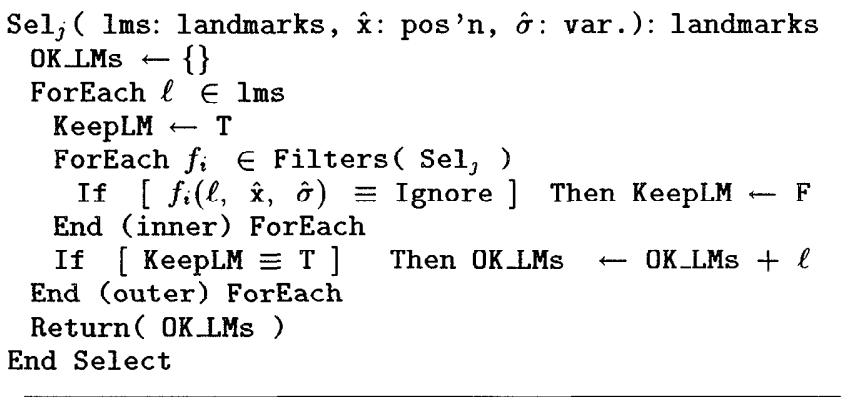

Figure 1: PseudoCodc for $\mathrm{Scl}_{j}$ Selection Function

where Type $(\ell)$ refers to the type of the landmark $\ell$, which can be "Door", "BlackStrip", etc. ${ }^{5}$ The parameter $K_{3}$ specifies the subset of landmark-types that should be used. Using "Posn $(\ell)$ " to refer to $\ell$ 's realworld coördinates and "AngleWidth $(\ell, \hat{\mathbf{x}})$ " to refer to the angle subtended by the landmark $\ell$, when viewed from $\hat{\mathbf{x}}$, TooSmall To $_{1}, k_{2}(\ell, \hat{\mathbf{x}}, \hat{\sigma})$ rejects the landmark $\ell$ if $\ell$ is both too far away (greater than $k_{1}$ meters) and also too small (subtends an angle less than $k_{2}$ degrees), from $R$ 's estimated position $\hat{\mathbf{x}}$.

Using these filters, $\mathcal{S}=\left\{\mathrm{Sel}_{k_{1}, k_{2}, K_{3}}\right\}$ is the set of all selection functions, over a combinatorial class of settings of these three parameters. As stated above, we want to find the best settings of thcse variables, which minimize the expected error AveErr $\left[\mathrm{Sel}_{k_{1}, k_{2}, K_{3}}\right]$.

Hill-Climbing in Uncertain Space: There are two obvious complications with our task of finding this optimal setting: First, as noted above, the error function depends on the distribution of situations, which is not known initially. Secondly, even if we knew that information, it is still difficult to compute the optimal parameter setting, as the space of options is large and ill-structured (e.g., $K_{3}$ is discrete, and there are subtle non-linear effects as we alter $k_{1}$ and $k_{2}$ ).

We use a standard hill-climbing approach to address the second problem, based on a set of operators $\mathcal{T}=\left\{\tau_{k}\right\}$ that each map one selection function to another; i.e., for each $s \in \mathcal{S}, \tau_{k}(s) \in \mathcal{S}$ is another selection function. We use the obvious set of operators: $\tau_{1}^{+}$increments the value of $k_{1}$ and $\tau_{1}^{-}$decrements $k_{1}$ 's value; hence $\tau_{1}^{+}\left(\operatorname{Sel}_{5,8},\{t 1, t 3, t 7\}\right)=\operatorname{Sel}_{6,8},\{t 1, t 3, t 7\}$ and $\tau_{1}^{-}\left(\operatorname{Sel}_{5}, 8,\{t 1, t 3, t 7\}\right)=\mathrm{Sel}_{4,8},\{t 1, t 3, t 7\}$. Similarly, $\tau_{2}^{+}$and $\tau_{2}^{-}$respectively increment and decrement the $k_{2}$ value. Therc arc 9 different $\tau_{3}^{i}$ operator, each of which "flips" the $i^{\text {th }}$ bit of $K_{3}$;

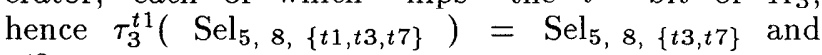
$\tau_{3}^{t 8}\left(\operatorname{Sel}_{5,8,\{t 1, t 3, t 7\}}\right)=\operatorname{Sel}_{5,8,\{t 1, t 3, t 7, t 8\}}$.

To address the first problem - viz., that the distribution is unknown -- we use a set of observed examples

\footnotetext{
${ }^{5}$ The current system contains nine different types: Miscellaneous, Black_Strip, Concave_Corner, Convex_Corner, Dark_Door, Light_Door, Picture, FireExtinguisher and Support_between_Windows.
} 


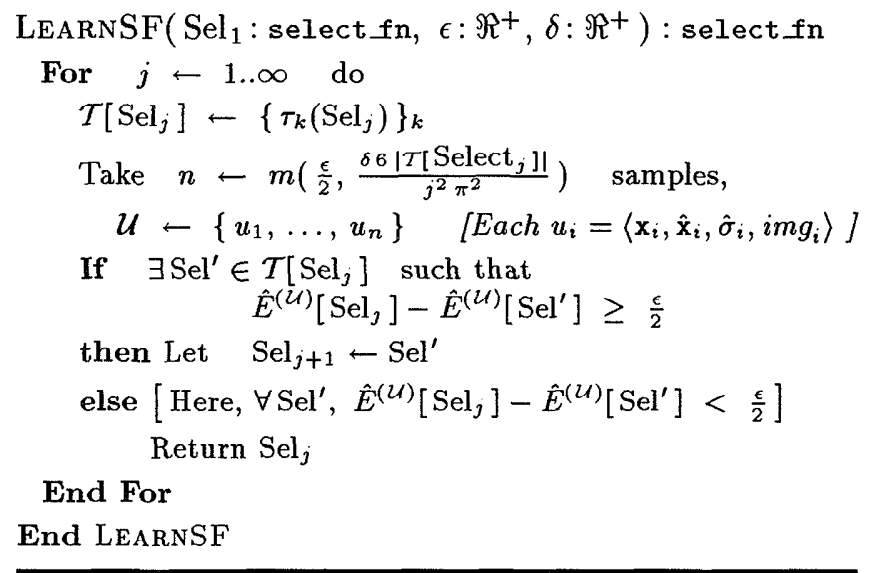

Figure 2: (Simplified) PseudoCode for LEARNSF

to estimate the relevant information: Let

$$
\begin{aligned}
\hat{E}_{k_{1}, k_{2}, K_{3}}^{(\mathcal{U})} & =\hat{E}^{(\mathcal{U})}\left[\operatorname{Err}\left(\operatorname{Sel}_{k_{1}, k_{2}, K_{3}}\right)\right] \\
& =\frac{1}{|\mathcal{U}|} \sum_{u_{i} \in \mathcal{U}} \operatorname{Err}\left(\operatorname{Sel}_{k_{1}, k_{2}, K_{3}}, u_{i}\right)
\end{aligned}
$$

be the empirical average error of the selection function $\mathrm{Sel}_{k_{1}, k_{2}, K_{3}}$ over the set of training samples $\mathcal{U}=$ $\left\{\left\langle\mathbf{x}_{i}, \hat{\mathbf{x}}_{i}, \hat{\sigma}_{i}, \mathrm{img}_{i}\right\rangle\right\}_{i}$, which we assume to be independent and identically distributed. We then use some statistical measure to relate the number of samples seen, to our confidence that $\hat{E}_{i}^{(\mathcal{U})}$ will be close to the real mean $\mu_{i}=E_{u_{j}}\left[\operatorname{Err}\left(\operatorname{Sel}_{i}, u_{j}\right)\right]=\operatorname{AveErr}\left(\operatorname{Sel}_{i}\right)$ value. In particular, we need a function $m(\cdots)$ such that, after $m(\epsilon, \delta)$ samples, we can be at least $1-\delta$ confident that the empirical average $\hat{E}^{(\mathcal{U})}$ will be within $\epsilon$ of the population mean $\mu$; i.e., $|\mathcal{U}| \geq m(\epsilon, \delta) \Rightarrow$ $\operatorname{Pr}\left[\left|\hat{E}^{(\mathcal{U})}-\mu\right|>\epsilon\right] \leq \delta$. If we can assume that the underlying distribution of error values is close to a normal distribution, then we can use

$$
m_{N o r m}(\epsilon, \delta)=\left(\frac{\lambda}{\epsilon} z^{-1}\left(1-\frac{\delta}{2}\right)\right)^{2}
$$

where the $z(p)=\frac{1}{\sqrt{2 \pi}} \int_{-\infty}^{p} e^{-\frac{x^{2}}{2}} d x$ function computes the $p^{\text {th }}$ quantile of the standard normal distribution $\mathcal{N}(0,1)$ (Bickel \& Doksum 1977).

The LEARNSF algorithm, sketched in Figure 2, ${ }^{6}$ combines the ideas of hill-climbing with statistical sampling: Given an initial selection function $\mathrm{Sel}_{1}=$ Sel $_{k_{1}, k_{2}, K_{3}} \in \mathcal{S}$, and the parameters $\epsilon$ and $\delta$, LEARNSF will use a sequence of example situations $\left\{u_{i}\right\}$ to climb from the initial $\mathrm{Sel}_{1}$ through successive neighboring selection functions $\left(\mathrm{Sel}_{1}, \mathrm{Sel}_{2}, \mathrm{Sel}_{3}, \ldots\right.$ ) until reaching, and returning, a final $\mathrm{Sel}_{m}$. With high probability, this $\mathrm{Sel}_{m}$ is essentially a local optimum. Moreover, LEARNSF requires relatively few samples for each climb. To state this more precisely:

${ }^{6}$ We actually use much more efficient, but more complex, algorithm that, for example, decides whether to climb

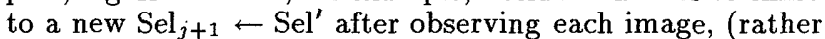
than a batch of $n$ images); see (Greiner \& Isukapalli 1994; Greiner 1994).

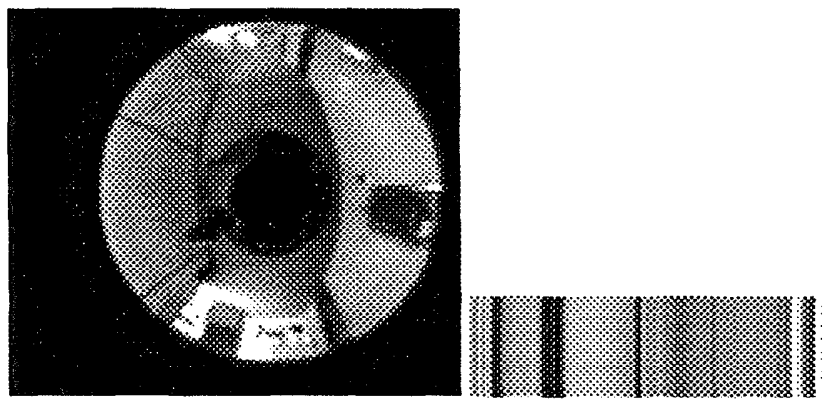

Figure 3: RATBOT's view (looking up at tree ornament), and "strip", corresponding to annulus in image

Theorem 1 (from (Greiner \& Isukapalli 1994))

The LEARNSF $\left(\mathrm{Sel}_{1}, \epsilon, \delta\right)$ process incrementally produces a serics of selection functions $\mathrm{Scl}_{1}, \mathrm{Sel}_{2}, \ldots, \mathrm{Scl}_{m}$, such that each $\mathrm{Sel}_{j+1}=\tau_{j}\left(\mathrm{Sel}_{j}\right)$ for some $\tau_{j} \in \mathcal{T}$ and, with probability at least $1-\delta$

1. the expected error of each selection function is strictly better than its predecessors i.e.,

$\forall 2 \leq j \leq m: A v e E r r\left(\operatorname{Sel}_{3-1}\right)<\operatorname{AveErr}\left(\operatorname{Sel}_{j}\right) ;$ and

2. the final selection function (returned by LEARNSF), Sel $l_{m}$, is an " $\epsilon$-local optimum" - i.e.,

$\neg \exists \tau \in \mathcal{T}: \operatorname{AveErr}\left(\tau\left(S e l_{m}\right)\right)<\operatorname{AveErr}\left(S e l_{m}\right)-\epsilon$.

given the statistical assumption that the underlying distribution is essentially normal. Moreover, LEARNSF will terminate with probability 1 , and will stay at any $\mathrm{Sel}_{j}$ (before either terminating or climbing to a new $S e l_{3+1}$ ) for a number of samples that is polynomial in $\frac{1}{\epsilon}, \frac{1}{\delta},|\mathcal{T}|$ and $\lambda=\max _{\tau \in \mathcal{T}}, \operatorname{Sel}_{\in S, u}|\operatorname{Err}(\operatorname{Sel}, u)-\operatorname{Err}(\tau(\operatorname{Sel}), u)|$, which is the largest difference in error between a pair of neighbor. ing selection functions for any sample.

\section{Empirical Results}

To test the theoretical claims that a good selection function can help an autonomous agent to register its position efficiently and accuratcly, and also that LEARNSF can help find such good selection functions, we implemented various selection functions and the LEARNSF learning algorithm, and incorporated them within the implemented autonomous agent, RATBOT, described in (Hancock \& Judd 1993). This section describes our empirical results.

We first took a set of 270 "pictures" at known locations within three halls of our building. Each of these pictures is simply an array of 360 intensity values, each corresponding to the intensity at a particular angle, in a plane parallel to the floor; these are shown on righthand side of Figure $3 .^{7}$ We have also identified 157 different landmarks in these regions, each represented as

\footnotetext{
${ }^{7}$ These were obtained using a "NOMAD 200 " robot with a CCD camera mounted on top, pointing up at a spherical mirror (which is actually a christmas tree ornament); see left-hand side of Figure 3. We then extract from this image a 1-pixel annulus, which corresponds to the light intensity at a certain height; see right-hand side of Figure 3 .
} 


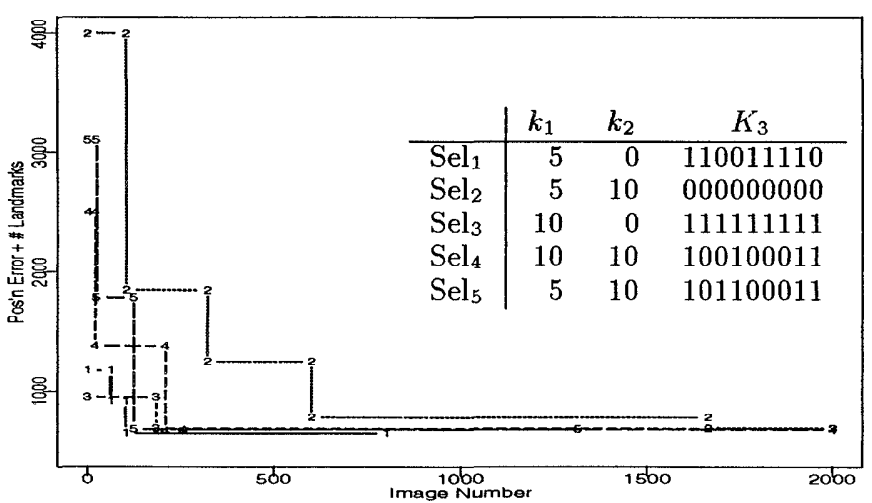

Figure 4: LEARNSF's Hill-Climbs, for different initial Selection Functions

simply an object of a specified type (one of the nine categories), located between a pair of coördinates $\left\langle x_{1}, y_{1}\right\rangle$ and $\left\langle x_{2}, y_{2}\right\rangle$; where, once again, this $\langle x, y\rangle$ plane is parallel to the floor and goes through the center of the bulb.

Each experiment used a particular initial selection function, error function, values for $\epsilon$ and $\delta$, way of estimating $R$ 's position, and statistical assumption. We first describe one experiment in detail, then discuss a battery of other experiments that systematically vary the experimental parameters.

Experiment\#1 Specification: LEARNSF began with the Sel $_{1}$ selection function shown in Figure 4. This function rejects a landmark if either it is more than 5 meters away from our estimated position and also subtends an angle less than 0 degrees, ${ }^{8}$ or if the landmark's type is one of Concave_Corner, Convex_Corner, or Support_between_Windows (these are the second, third and ninth types, corresponding to the bits that are 0 in the $\mathrm{Sel}_{1}$ row of Figure 4). We used $\delta=0.05$, meaning that we would be willing to accept roughly 1 mistake in 20 runs. The $\epsilon=0.1$ setting means that we do not care if the average error of two selection functions differs by less than $0.1 \mathrm{~m}$; as we allowed errors as large as $4 \mathrm{~m}$, this corresponds to an allowable tolerance of only $2.5 \%$.

As our goal is to minimize both positional error and computational time, we use an error function that is the weighted sum of the positional error (which is the difference between the obtained estimated position and the real position) and “\#landmarks-to-pos'n-error ratio" times the number of landmarks that were selected. Here, we set the ratio to 0.01 , to mean, in effect, that each additional landmark "costs" $0.01 \mathrm{~m}$.

Finally, while we know that image img $g_{i}$ is taken at location $\mathbf{x}_{i}$, it unrealistic to assume that RATBOT will know that information; in general, we assume that RATBOT will instead see an approximate $\hat{\mathbf{x}}_{i}$. We

${ }^{8}$ As nothing can subtend an angle strictly less than $0^{\circ}$, this first clause is a no-op - i.e., it will not reject any landmark.

\begin{tabular}{ccc} 
Sample \# & Selection Function & $E[$ LM-Err $]$ \\
\hline 0 & Sel $_{1}=\langle\langle 5,0\rangle ;[110011110]\rangle$ & 1.171 \\
62 & Sel $_{1: 1}=\langle\langle 5,0\rangle ;[110111110]\rangle$ & 0.916 \\
102 & Sel $_{1: 2}=\langle\langle 5,2\rangle ;[110111110]\rangle$ & 0.647
\end{tabular}

Table 1: Data for LearnSF's Climbs from $\mathrm{Sel}_{1}$ model this by setting $\hat{\mathbf{x}}_{i}=x_{i}+\nu_{i}^{(\sigma)}$, where each $\nu_{i}^{(\sigma)}$ is a normally-distributed random value with mean zero and variance $\sigma$. Here, we used $\sigma=0.3 \mathrm{~m}$. Recall also that the Locate function needs a value for $\hat{\sigma}$ to constrain its landmark-location process; we also set $\hat{\sigma}$ to be $\sigma$.

Experiment\#1 Results: Given these settings, LEARNSF observed 62 labeled samples before climbing to the new selection function $\mathrm{Sel}_{1: 1}=$ $\langle\langle 5,0\rangle ;[110111110]\rangle$, which differs from Sel $_{1}$ only by not rejecting all Convex_Corners. ${ }^{9}$ It continued using this selection function for 40 additional samples, before climbing to the $\operatorname{Sel}_{1: 2}=\langle\langle 5,2\rangle$; [110111110] $\rangle$ sclection function, which rejects landmarks that are both more than $5 \mathrm{~m}$ from $R$ 's estimated position and also less than $2^{\circ}$. It continued using this $\mathrm{Sel}_{1: 2}$ function for another 700 samples before LEARNSF terminated, dcclaring this selection function to be a "0.1-local op-

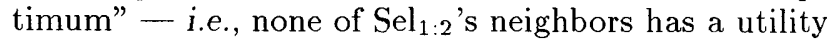
score that is more than $\epsilon=0.1$ better than $\mathrm{Sel}_{1: 2}$. (We found, in fact, that $\mathrm{Sel}_{1: 2}$ is actually a bona fide local optimum, in that none of its neighbors is even as good as it is.)

The solid line (labeled "1") in Figure 4 shows LEARNSF's performance here. Each horizontal linesegment corresponds to a particular selection function, where the line's $y$-value indicates the "average test error" of its selection function, which was computed by running this selection function through all 270 images. ${ }^{10}$ These horizontal lines are connected by vertical lines whose $x$-value specify the sample number when LEARNSF climbed. Table 1 presents a more detailed break-down of this data.

Other Variants: Our choice of $\mathrm{Sel}_{1}=\langle\langle 5,0\rangle$; [110111110] 〉 was fairly arbitrary; we also considcred the four other reasonable starting selection functions shown in Figure 4. Notice that $\mathrm{Sel}_{2}=$ $\langle\langle 5,10\rangle ;[000000000]\rangle$ rejects every landmark; and $\mathrm{Sel}_{3}=\langle\langle 5,0\rangle ;[111111111]\rangle$ accepts every landmark. Figure 4 also graphs the performance of these functions. Notice that LEARNSF finds improvements in all five cases.

We also systematically varied the other parameters: trying values of $\epsilon=1.0,0.2,0.1,0.05,0.02,0.01$, $0.005 ; \delta=0.005, \quad 0.01,0.05,0.1 ; \sigma=$

\footnotetext{
${ }^{9} \mathrm{Sel}_{i: j}$ refers to the selection function reachcd after $j$ climbs, when starting from Sel ${ }_{i}$. Hence, Sel $_{i: 0} \equiv \operatorname{Sel}_{i}$.

${ }^{10}$ To avoid testing on the training data, we computed this value using a new set of randomly-generated positional

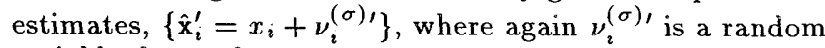
variable drawn from a 0 -mean $\sigma$-variance distribution.
} 
$0,0.3,0.5,1.0$; and the "\#landmark-to-pos'n-error ratio" of $0,0.02,0.05,0.1,0.2$. (The 0 setting tells LEARNSF to consider only the accuracy of a landmark set, and not the cost of finding those landmarks.) We

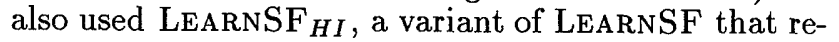
places the $m_{N o r m}(\cdot)$ function with the weaker

$$
m_{H I}(\epsilon, \delta)=\frac{1}{2}\left(\frac{\lambda}{\epsilon}\right)^{2} \ln \frac{2}{\delta}
$$

function, which is based on Hoeffding's inequality (Hoeffding 1963; Chernoff 1952), and so does not require the assumption that the error values are normally distributed. All of these results are reported, in detail, in (Greiner \& Isukapalli 1994).

Summary of Empirical Results: The first obvious conclusion is that selection functions are useful; notice in particular that the landmarks they returned enabled $R$ to obtain fairly good positional estimates - within a few tenths of a meter. Notice also that the obvious degenerate selection function, $\mathrm{Sel}_{3}$ which accepted all landmarks, was not optimal; i.e., there were functions that worked more effectively. Secondly, this LEARNSF function works effectively, as it was able to climb to successively better selection functions, in a wide variety of situations. Not surprisingly, we found that the most critical parameter was the initial selection function; the values of $\epsilon, \delta, \sigma$ and even the "\#landmark-topos'n-error ratio" had relatively little effect. We also found that this LEARNSF Norm $_{\text {system seemed to work }}$ more effectively than the version that did not require the normality assumption, LEARNSF ${ }_{H I}$ : in almost all instances, both systems climbed through essentially the same selection functions, but LEARNSF Norm $_{\text {re- }}$ quired many fewer samples - by a factor of between 10 and 100 ! (In the numerous different runs that used $\delta=0.05$, LEARNSF $_{N o r m}$ climbed a total of 84 times and terminated 24 times, and so had $84+24=108$ opportunities to make a mistake; it made a total of only 3 mistakes, all very minor.) Finally, LEARnSF's behavior was also (surprisingly) insensitive to the accuracy of $R$ 's estimated position, over a wide range of errors; e.g., even for non-trivial values of $|x-\hat{\mathbf{x}}|$.

\section{Conclusion}

While there are many techniques that use observed landmarks to identify an agent's position, they all depend on being able to effectively find an appropriate set of landmarks, and will produce degraded or unacceptable information if the landmarks arc not found, or mis-identified. We can avoid this problem by using only the subset of "good" landmarks. As it can be very difficult to determine this subset a priori, we present an algorithm, LEARNSF, that uses a set of training samples to learn a function that selects the appropriate subset of the landmarks, which can then be used robustly to determine our agent's position. We then prove that this algorithm works effectively - both theoretically and empirically, based on real data obtained using an implemented robot.

\section{Acknowledgments}

We gratefully acknowledge the help we received from Thomas Hancock, Stephen Judd, Long-Ji Lin, Leonid Gurvits and the other members of the RatBOT team.

\section{References}

Bickel, P. J., and Doksum, K. A. 1977. Mathematical Statistics: Basic Ideas and Selected Topics. Oakland: Holden-Day, Inc.

Case, M. 1986. Single landmark navigation by mobile robots. In SPIE, volume $727,231-38$.

Chernoff, H. 1952. A measure of asymptotic efficiency for tests of a hypothesis based on the sums of observations. Annals of Mathematical Statistics 23:493-507.

Cox, I., and Wilfong, G., eds. 1990. Autonomous Robot Vehicles. Springer-Verlag.

Engelson, S. P. 1992. Active place recognition using image signatures. In SPIE Symposium on Intelligent Robotic Systems, Sensor Fusion V, 393-404.

Fennema, C.; Hanson, A.; Riseman, E.; Beveridge, J.; and Kumar, R. 1990. Model-directed mobile robot navigation. IEEE Transactions on Systems, Man and Cybernetics 20(6):1352-69.

Greiner, R., and Isukapalli, R. 1994. Learning to select useful landmarks. Technical Report SCR-LS94-473.

Greiner, R. 1994. Probabilistic hill-climbing: Theory and applications. Technical report, SCR.

Gurvits, L., and Betke, M. 1994. Robot navigation using landmarks. Technical Report SCR-94-TR-474, SCR/MIT. Hancock, T., and Judd, S. 1993. Ratbot: Robot navigation using simple visual algorithms. In 1993 IEEE Regional Conference on Control Systems.

Hancock, T., and Judd, S. 1994. Hallway navigation using simple visual correspondence algorithms. Technical Report SCR-94-TR-479, SCR.

Hoeffding, W. 1963. Probability inequalities for sums of bounded random variables. Journal of the American Statistical Association 58(301):13-30.

Kosaka, A., and Kak, A. C. 1992. Fast vision-guided mobile robot navigation using model-based reasoning and prediction of uncertainties. Computer Vision, Graphics, and Image Processing 56(3):271-329.

Kuipcrs, B. J., and Byun, Y.-T. 1988. A robust, qualitative method for robot spatial learning. In $A A A I-88$, 774-79.

Kuipers, B. J., and Levitt, 'I. S. 1988. Navigation and mapping in large-scale space. AI Magazine 9(2):25-43.

Levitt, T. S., and Lawton, D. T. 1990. Qualitative navigation for mobile robots. Artificial Intelligence 44:305-60.

Smith, R., and Cheeseman, P. 1987. On the representation and estimation of spatial uncertainty. International Journal of Robotics Research 5(4):56-68.

Sugihara, K. 1987. Location of a robot using sparse visual information. Robotics Research: The Fourth International Symposium, 319-26. MIT Press.

Sugihara, K. 1988. Some location problems for robot navigation using a single camera. Computer Vision, Graphics and Image Processing 42(1):112-29. 